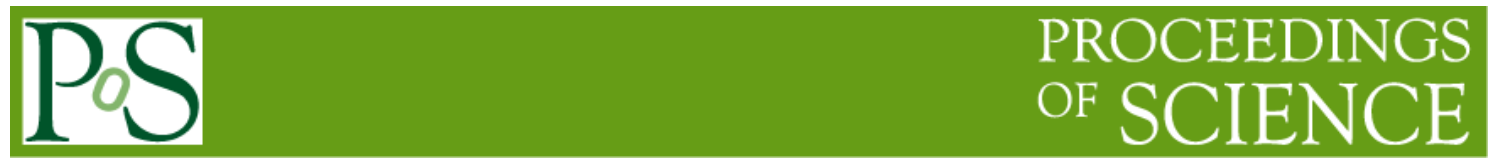

\title{
New Boson (Higgs?) into yY at CERN
}

\section{V.Roinishvili}

JINR,

Dubna, Moscow Region, Russia

EAIP

Tamarashvili,6, Tbilisi, Georgia

E-mail: roinishvilieihep.ru

A very simple analyse of the combined data of ATLAS and CMS, published in PL B 716 (2012) on discovery of a new boson which decays into two photons Is presented. The obtained total statistical significance of the signal is $7.5 \pm 1$ standard deviations with the mass located at $125.6 \pm 0.3 \mathrm{GeV}$.

LHC on the March - IHEP-LHC

20-22 November 2012

Institute for High Energy Physics, Protvino,Moscow region, Russia 
I think that one day somebody will combine the CMS and ATLAS data on the discovery of a new boson $\mathrm{B}$ (Higgs?) $\rightarrow \gamma \gamma$. But I am more than 80 years old and have no time to wait. So I decided to do it now using already published ATLAS and CMS articles in PL B 716 (2012). Because in the articles there are no tables with raw data neither of CMS, nor of ATLAS, together with Nikolai Tkachenko we have digitized figures from the ATLAS (fig.4) and from the CMS (fig.3). Obtained in this way data were fitted (using MINUIT) by the following expression:

$$
Y=\frac{W}{\sqrt{2 \pi} \sigma} \exp \left[-\frac{\left(m_{W}-M\right)^{2}}{2 \sigma^{2}}\right]+B k g
$$

where $\mathrm{Y}$ corresponds to the $\mathrm{S} /(\mathrm{S}+\mathrm{Bkg})$ weighted events from Fig.3 in the CMS article or to the $\sum$ weights from Fig.4 in the ATLAS article. W, M and $\sigma$ are the weight, mass and width of the signal. The shape of the background was chosen as

$$
B k g=\frac{1 .}{\left(A+B m_{\gamma}\right)^{4}}
$$

which describes the backgrounds very well for both experiments and has only 2 parameters. There are 4 free parameters - W and $\mathrm{M}$ for the signals and A and B for the backgrounds. The resolutions of the effective mass of two gammas $-\sigma$ where fixed to the known values of the spectrometers.
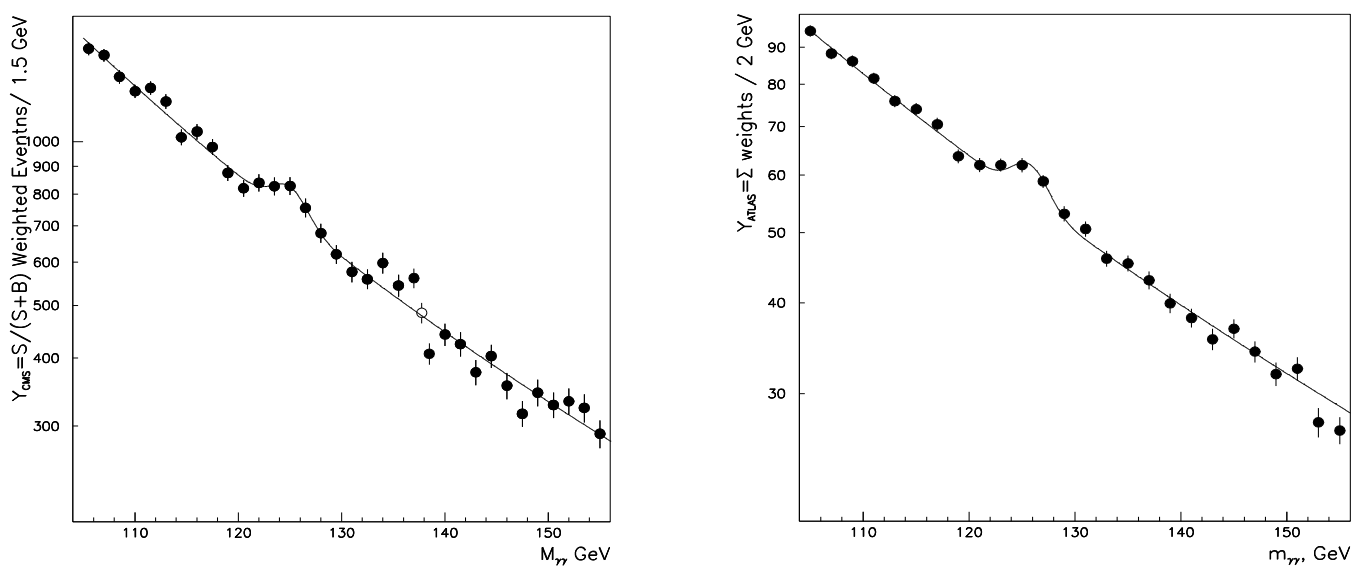

$$
\begin{aligned}
& \text { CMS } \\
& W=423 \pm 99 \\
& M=125.0 \pm 0.5 \mathrm{GeV} \\
& A=0.015 \\
& B=-0.0017 \\
& N S T D V=W / \Delta W=4.3 \pm 1
\end{aligned}
$$

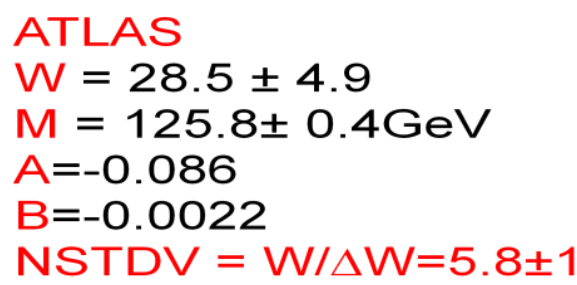

The obtained parameters of the backgrounds were used to define the ratio $\mathrm{R}=\mathrm{Y} / \mathrm{Bkg}$ for $\mathrm{CMS}$ and ATLAS, which are plotted on the following picture. 


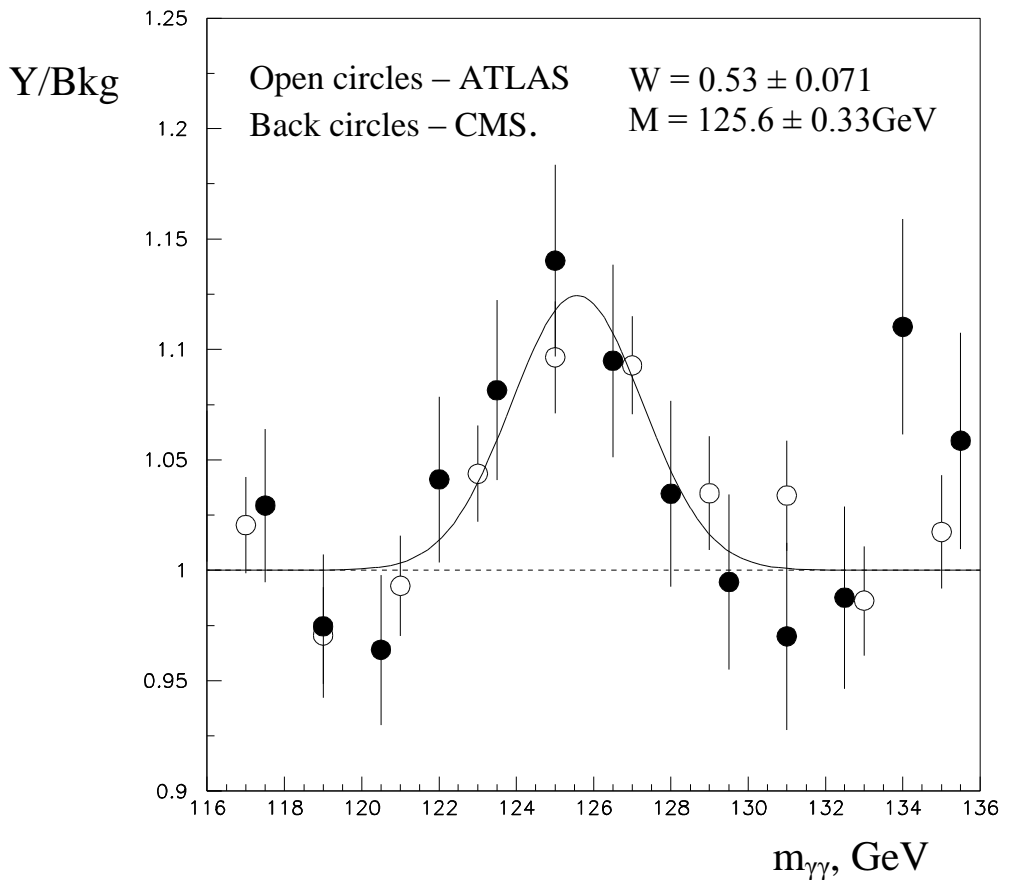

Fit $: Y / B k g=\frac{W}{\sqrt{2 \pi} \sigma} \exp \left[-\frac{\left(m_{r}-M\right)^{2}}{2 \sigma^{2}}\right]+1$.

$\chi^{2} / \mathrm{ndf}=17.5 / 21$

Fit was done with two free parameters - the weight $\mathrm{W}$ and the mass of the new Boson M. $\sigma$ is fixed to the values of the CMS and ATLAS resolution. The significance of the observed signal is defined as a number of standard deviations, NSTDV $=\mathrm{W} / \Delta \mathrm{W}$, where $\Delta \mathrm{W}$ is the error of $\mathrm{W}$ obtained by MINUIT.

We obtain $\mathrm{M}=125.6 \pm 0.3 \mathrm{GeV}$ and $\mathrm{NSTDV}=7.5 \pm 1$ for the published in $2012 \mathrm{CERN}$ data.

I would like to thank V.Kabachenko for his technical help in preparation of this report . 